\title{
Pierre Ducassé et la revue Structure et Évolution des Techniques (1948-1964)
}

Pierre Ducassé and the journal Structure et Évolution des Techniques

(1948-1964)

Ronan Le Roux

\section{OpenEdition}

12 Journals

Édition électronique

URL : http://journals.openedition.org/dht/1761

DOI : $10.4000 /$ dht. 1761

ISSN : 1775-4194

Éditeur :

Centre d'histoire des techniques et de l'environnement du Cnam (CDHTE-Cnam), Société des élèves du CDHTE-Cnam

Édition imprimée

Date de publication : 1 décembre 2011

Pagination : 119-134

ISBN : 978-2-9530779-7-1

ISSN : 0417-8726

\section{Référence électronique}

Ronan Le Roux, «Pierre Ducassé et la revue Structure et Évolution des Techniques (1948-1964)»,

Documents pour I'histoire des techniques [En ligne], 20 | 2e semestre 2011, mis en ligne le 17 septembre 2012, consulté le 10 décembre 2020. URL : http://journals.openedition.org/dht/1761 ; DOI : https:// doi.org/10.4000/dht.1761 


\title{
Pierre Ducassé et la revue Structure et Évolution des Techniques (1948-1964)
}

\author{
Ronan Le Roux \\ CETCOPRA / Université Paris 1 Panthéon-Sorbonne
}

\begin{abstract}
Résumé
Période d'intense modernisation comme d'interrogations passionnées sur les conséquences du progrès, les années cinquante ont manifesté un certain foisonnement d'approches intellectuelles consacrées aux techniques. En s'attardant dans les bibliographies françaises concernées, on croise parfois le titre d'une revue introuvable : Structure et Évolution des Techniques (S.É.T.). Cette publication est l'initiative de Pierre Ducassé (1905-1983), philosophe et historien des sciences et des techniques, qui fut d'abord secrétaire de l'Institut d'Histoire des Sciences, professeur de philosophie à la Faculté des Lettres de Besançon, puis professeur au Conservatoire national des arts et métiers. Dans cet article, on se propose de voir comment le projet éditorial S.É.T. et la trajectoire intellectuelle (et personnelle) de Ducassé s'éclairent réciproquement. Selon notre lecture, le thème des techniques devient progressivement, pour ce spécialiste d'Auguste Comte, l'occasion de développer une interrogation critique à l'égard de l'héritage positiviste. Créer une revue permet alors de se donner un lieu pour accueillir des points de vue novateurs de réflexion sur les techniques contemporaines, notamment autour de la cybernétique telle qu'elle est reçue et ré-élaborée dans le contexte hexagonal de l'époque.
\end{abstract}

Résumés et mots clés en anglais sont regroupés en fin de volume, accompagnés des mots clés français

\section{e positivisme à l'épreuve des techniques \\ LPierre Ducassé découvre la philosophie durant} sa licence de sciences à la Sorbonne, et décide de s'y consacrer. II commence, sous la direction d'Abel Rey, une thèse portant sur la méthode philosophique d'Auguste Comte. Rey fonde l'Institut d'Histoire des Sciences de Paris en 1932, et choisit d'y placer Ducassé au poste de secrétaire. Ducassé, qui occupera le poste jusqu'à l'arivée de Canguilhem en 1955, prend en charge une part importante de l'organisation. ॥ entretient par ailleurs une certaine proximité avec le milieu positiviste : au-delà de sa thèse, pour laquelle il sollicite notamment l'avis régulier de Paul Edger, on le trouve par exemple en 1936 prononçant l'allocution en l'honneur du soixante-dix-neuvième anniversaire de la mort de Comtel. II soutient en 1938 sa Thèse principale

1 Publiée dans la Revue Positiviste Internationale sous le titre "Le témoignage d'Auguste Comte et le réveil des espérances positives $\%$. sous le titre Méthode et intuition chez Auguste Comte, et sa Thèse complémentaire sous le titre Essai sur les origines intuitives du positivisme. Les deux Thèses sont publiées l'année suivante, chez Alcan et aux Presses Universitaires de France. On trouve des compte-rendus élogieux de ses travaux aussi bien dans Thalès, la revue de l'IHST, que dans la Revue Positiviste Internationale ${ }^{2}$. Dans un article sur les débuts de l'IHST, Jean-François Braunstein évoque les tentatives des positivistes pour se rapprocher de l'Institut ${ }^{3}$. On peut supposer qu'Edger comptait pour cela sur Ducassé, qu'il appelle son "cher correligionnaire ".

\footnotetext{
2 Voir le compte-rendu de Hélène Metzger dans le tome IV de Thalès (1937-39), et celui de G. Grimanelli dans la Revue Positiviste Internationale de janvier $1936\left(n^{\circ} 1\right)$.

3 Jean-François Braunstein, "Abel Rey et les débuts de I'Institut d'Histoire des Sciences et des Techniques ॥, dans Michel Bitbol \& Jean Gayon, Épistémologie française, Paris, PUF, 2006, pp. 173-191.
} 
Mais la continuité n'est pas totale entre Ducassé et le positivisme, ni socialement, ni intellectuellement. Le projet d'Edger de transférer les « reliques ॥ positivistes (les manuscrits de Comte) à l'IHST n'aboutit pas. Bien que ce soit Rey qui maîtrise les critères administratifs de la décision, le ton que le secrétaire emploie dans la fin de non-recevoir adressée à Edger suggère bien qu'il n'est pas le "cher correligionnaire " qu'espérait ce dernier ${ }^{4}$. L'importance que sa thèse accorde à l'intuition dans l'ensemble de l'œuvre comtienne n'aurait par ailleurs pas fait l'unanimité en Sorbonne. Mais c'est sur un autre terrain que Ducassé va creuser philosophiquement un écart avec l'héritage positiviste : celui des techniques. S'il n'est malheureusement pas possible de déterminer avec certitude la façon dont ce thème est devenu privilégié pour lui, on pourra se plaire à interpréter, dans l'allocution funéraire de 1936, que c'est dans "les bouleversements objectifs et subjectifs qu'entraîne le progrès technique » que Ducassé voit déjà la limite historique de la pensée de Comte ${ }^{5}$ ce qui peut sembler bien paradoxal à l'égard de cet élève de Saint-Simon. On peut ainsi, moyennant la circonspection qu'appelle toute enquête rétrospective, déceler dans cet hommage à Comte une intuition de jeunesse annonçant la réflexion critique qui va se déployer explicitement dans les années cinquante. On ne peut que supposer, faute de traces écrites (les archives personnelles de Ducassé ont brûlé dans l'incendie d'un gardemeuble), que dès les années trente, Ducassé est sensible à une certaine réalité contemporaine des manifestations techniques qui met en défaut le système de philosophie positive, une réalité pour laquelle le système n'a pas de réponse, théorique

\footnotetext{
4 Lettre du 29 juin 1938 : « Nous respectons entièrement le caractère que vous attachez aux manuscrits d'Auguste Comte et aux documents iconographiques dans leur totalité, mais si, dans les conditions précitées, il ne vous semble pas possible de les confier à l'Institut d'Histoire des Sciences et des Techniques, il est à notre avis inutile de poursuivre les pourparlers pour l'ensemble du dépôt ॥. Ce n'est pas là un propos excessivement imprégné d'idolâtrie ou de regret à l'égard des « reliques » (le terme est d'Edger lui-même).

5 Pierre Ducassé, "Le témoignage d'Auguste Comte et le réveil des espérances positives $॥$, Revue Positiviste Internationale, 31e année, nov. 1936, n 5-6, p. 6. C'est cette limitation qui doit s'avérer instructive aux yeux de Ducassé : "L'édifice d'Auguste Comte n'aurait pas été, intellectuellement, la cible de choix des destructions contemporaines, s'il n'avait inclus quelque intuition très exigeante de l'ordre humain. L'œuvre fut détruite par le siècle, mais elle porte par là même un témoignage incisif sur le siècle, et sur l'humanité : Son langage n'est pas ambigu ॥ (ibid., p. 5).
}

ou pratique. Si l'on ne peut plus comprendre les techniques, c'est que celles-ci ne découlent pas de la Théorie. Le positivisme serait en quelque sorte dépassé par le machinisme qu'il est pourtant supposé organiser, du moins aménager et en quelque sorte encadrer. Comment interpréter autrement un intérêt progressif pour l'étude historique des techniques, qui donne lieu, en 1945, à la publication du « Que saisje ? » intitulé Histoire des techniques? Cette vocation à connaître par-delà les catégories comtiennes (ou en-deçà d'elles), peut-être impulsée par les débats qui agitent alors les intellectuels français sur la question du machinisme ${ }^{6}$, coïncide avec les dix années comprises entre sa Thèse et le lancement de la revue S.É.T. en 1948. Durant ces dix ans, il écrit deux petits ouvrages de synthèse dans la collection «Que sais-je ? ॥: I'Histoire des techniques, et Les grandes philosophies, comme s'il s'agissait de faire un point de part et d'autre avant de déployer une pensée véritablement critique confrontant ces deux mondes.

Les catégories de la philosophie positive qu'il va s'agir d'interroger pour les subvertir, il en a exploré les déclinaisons dans sa Thèse :

«Les deux premiers textes [réunis en Appendice de la thèse] se rattachent immédiatement à l'idée directrice de notre recherche. Leur rapprochement doit, croyons-nous, laisser peu de doute sur le bien-fondé de la thèse que nous soutenons, savoir : que tout le système de Comte se construit sur l'intuition d'un rapport entre l'abstrait et le concret [... ${ }^{7}$ "l.

Cette dichotomie abstrait/concret, qui parcourt l'ensemble du système, se répercute à ses différents niveaux et structure donc aussi le statut de la technique dans l'optique positiviste. C'est là l'argument que Ducassé va articuler de façon explicite à partir du milieu des années cinquante pour son élaboration critique :

"On sait que la « clef de voûte » philosophique du positivisme, conçu comme harmonie nécessaire des méthodes de la science et comme intégration de la pensée scientifique dans l'histoire de la civilisation, réside dans la séparation générale entre théorie et pratique : distinction poussée, depuis le contraste de la pensée et de l'action, jusqu'à la distinction des

\footnotetext{
6 Voir par exemple Jacques Guillerme, préface à Jacques Lafitte, Réflexions sur la science des machines, $2^{\mathrm{e}}$ éd., Paris, Vrin, 1972.

7 Pierre Ducassé, Essai sur les origines intuitives du positivisme, Appendice, I, p. 229.
} 
sciences abstraites et des sciences concrètes - au-delà - jusqu'à la division politique du pouvoir en spirituel et temporel.

Or cette distinction transpose une notion propre aux économistes : la division du travail que Comte généralise (alors que Marxl'élabore dialectiquement). Dans cette généralisation, Comte est porté (et emporté) par l'éducation mathématique, polytechnicienne, dont sa jeunesse subit fortement l'empreinte, par son goût de l'enseignement mathématique, par l'esprit " pédagogique » des mathématiques appliquées à l'art de l'ingénieur ${ }^{8}$.

L'application de la théorie à la pratique fait l'objet des "sciences concrètes ", qui constituent l'activité d'une catégorie socio-culturelle dont Ducassé estime que c'est l'œuvre de Comte qui en annonce l'apparition historique et en reconnaît l'importance : les ingénieurs. Cependant, la place accordée à la technique dans le système positiviste reste insuffisante, et se dégrade même avec l'évolution de la pensée comtienne. Dans un premier temps, si les "sciences concrètes ॥ jouent bien leur rôle, c'est le statut de l'ingénieur qui révèle cette insuffisance :

"Classe intermédiaire ", doctrine " intermédiaire ", statut " moyen », le niveau de l'ingénieur se définit à partir de réalités qui le dépassent. L'homme qu'appelaient à la fois les progrès de la science et les besoins de l'industrie est conçu, par la philosophie scientifique du positivisme naissant, comme un compromis $(\ldots)^{9} \%$.

Par la suite, ce sont les "sciences concrètes" elles-mêmes qui disparaissent du système, et avec elles la dignité philosophique de la technique:

" En principe, la « catégorie technique » est donc présente à l'arrière-plan de toutes les constructions abstraites du positivisme, mais elle n'y figure jamais en titre propre, et, pour ainsi dire, comme pouvoir autonome. (...)

Au niveau de la technicité, (...) le contenu effectif des références concrètes subit une modification entre le début et la fin de l'entreprise comtienne :

8 Pierre Ducassé, " Auguste Comte et la Philosophie des Techniques $》$, S.É.T. n 55-56, p. 11.

9 Pierre Ducassé, Les techniques et le philosophe, Paris, PUF, 1958, collection « Philosophie de la matière », p. 61. modification reconnue et voulue par Comte, profondément conforme d'ailleurs à la logique de son œuvre (...).

En effet, au moment où Comte passe à l'effectuation sociologique, morale et religieuse des grandeurs concrètes impliquées par sa philosophie théorique, rien ne change en apparence de l'ordre de la connaissance et de ses rapports à l'action; tout change, en fait, parce que les méthodes d'action ne se rattachent plus exactement comme avant aux voies de la pensée abstraite. Sans être altéré dans sa fonction systématique générale, le rapport de la science à la technique est « réduit » par suppression d'une intermédiaire, antérieurement admis comme nécessaire, la science « concrète ».

Renoncer aux sciences concrètes, c'est, pour Comte, (...) renoncer à toute diversité foncière, à toute originalité spéciale des disciplines d'action, conçues comme systèmes relativement autonomes de complexes technico-scientifiques.

Conception profonde, propre à freiner la croissance abusive des «préjugés utilitaires», mais capable aussi de stériliser le développement des techniques et - par voie de récurrence le progrès des moyens de la science et peutêtre même toute l'exubérance créatrice des initiatives scientifiques ${ }^{10} \mathrm{l}$.

Dans son Cours, en effet, Comte estimait que :

" Le corps de doctrines propre à cette classe nouvelle [des ingénieurs], et qui doit constituer les véritables théories directes des différents arts, pourrait sans doute donner lieu à des considérations philosophiques d'un grand intérêt et d'une importance réelle. Mais un travail qui les embrasserait conjointement avec celles fondées sur les sciences proprement dites serait aujourd'hui tout à fait prématuré ; car ces doctrines intermédiaires entre la théorie pure et la pratique directe ne sont point encore formées ${ }^{11}$ ॥.

En sacrifiant la considération de "l'élément essentiel de l'action technique ॥, le positivisme brise le socle sur lequel il s'est construit, d'après Ducassé

10 Pierre Ducassé, " Auguste Comte et la Philosophie des Techniques ॥, S.É.T. n 55-56, pp. 13-14. C'est ce renoncement à la place intermédiaire et autonome de la science concrète qui, d'après Ducassé, fait diverger la politique positive du technocratisme saint-simonien.

11 Auguste Comte, Cours de Philosophie positive, t.1, Paris, Hermann, 1998, p.47. 
qui souligne « la relative fermeture de cette doctrine aux chances de rajeunissement intellectuel liées aux prodigieux développements des techniques issues de la science $11^{12}$.

Si l'évacuation de la consistance propre à la technique constitue le principal point d'achoppement du positivisme aux yeux de Ducassé, celui-ci n'en fait pas pour autant une particularité de la pensée comtienne : son ouvrage majeur de 1958, Les techniques et le philosophe, dresse un constat d'échec de la philosophie en général face aux techniques. Le ton n'est plus celui du chapitre "La philosophie moderne et la réhabilitation des techniques " de l'Histoire des techniques, où sont évoqués Bacon, Descartes, Galilée, Vinci et le sens pratique des encyclopédistes. C'est avec l'ère industrielle que la philosophie, qui dominait traditionnellement les autres domaines (sciences, art, religion), cesse de culminer. Mais si le thème de la Technique, traditionnellement secondaire, vient au-devant de la scène avec Marx, ce n'est qu'en tant qu'il est lié à d'autres problèmes jugés plus fondamentaux : le travail, la propriété, les conditions de production idéologiques. La Technique n'était vue qu'à travers ses manifestations. Le constat historique de son autonomisation - laquelle va de pair avec la déchéance de la philosophie - impose une remise en question radicale. La Technique « se joue » de la philosophie, et à cette autonomie correspond un vide de la pensée, une désertion ou un repli.

« Et, sans doute, le phénomène essentiel qui sensibilise présentement le philosophe à la technicité réside bien dans l'accélération générale des transformations pratiques issues de la science, et dans l'angoisse humaine qui s'y attache. La philosophie n'est pas indifférente au trouble universel : autre chose, même pour elle, et peut-être surtout pour elle, est d'ordonner un monde réputé stable, d'une cohérence merveilleusement indépendante de nous, ou de réfléchir sur un monde menacé de mort violente par la croissance déséquilibrée de nos plus savantes méthodes d'action.

Ce genre d'angoisse ne fait malheureusement que rendre plus proche à une raison lucide, mais sans prise apparente sur les forces qui la pressent, le sentiment de son infirmité ; le malaise spécifiquement philosophique de notre époque réside en cette évidence "aveuglante " d'où naît la plus redoutable tentation, qui

12 lbid., p. 15. est d'ériger en impuissance de droit, sous un masque d'indifférence hautaine, l'apparente impuissance de fait, ou, en sens inverse, de confondre la conduite de détachement, que suppose toute fonction philosophique, avec l'abdication inavovée. Une pensée qui croit annuler l'obstacle en l'ignorant, avant d'en avoir apprécié, puis assimilé la résistance, refuse son propre avenir.

Entre cette "ignorance qui s'ignore " ou dont la prétention philosophique ne vise les techniques qu'à travers une culture partiale, périmée, en tout cas inefficace, et la lucidité d'un refus "impossible " et incapable, malgré sa hauteur, d'annuler ce qu'il évite de connaître, quelle voie peut tenter la recherche du philosophe, dès qu'elle se montre soucieuse de définir, à leur niveau exact, le sens des techniques et la manière d'être du technicien ? ${ }^{13}$ ».

Ducassé en appelle donc - ainsi commence Les techniques et le philosophe - à « un type nouveau de recherches ॥. Si l'ouvrage de 1958 a pour vocation d'en estimer les conditions de possibilité philosophiques, on peut supposer que, dès 1948, c'est la revue Structure et Évolution des Techniques qui doit accueillir (et, peut-être, incarner) ce «type nouveau de recherches $"$.

\section{S.É.T., un lieu pour de nouvelles pistes}

Qu'y a-t-il donc à connaître des techniques en tant que telles, hormis les savoir-faire tenant à leur conception, leur utilisation et leur maintenance? C'est sans doute à la fin de l'Histoire des techniques que la réponse est la plus simplement formulée :

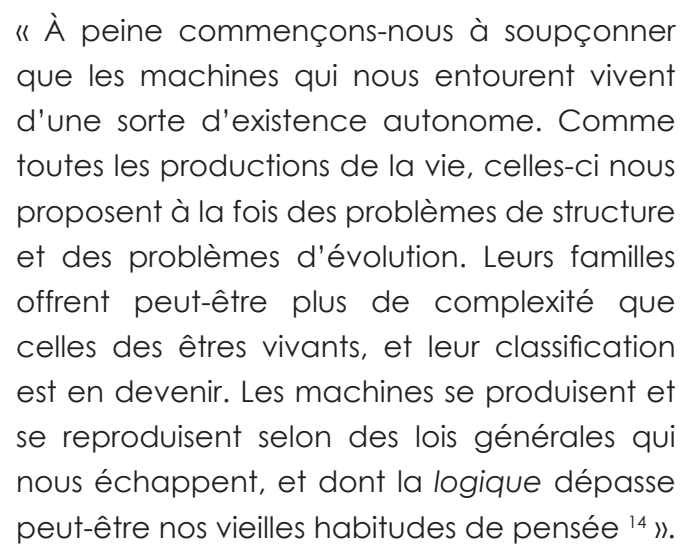

13 P. Ducassé, Les techniques et le philosophe, op. cit., pp. 12-13. 14 Pierre Ducassé, Histoire des techniques, PUF, 1945 (4e éd. 1958), collection « Que sais-je ? » n 126, p. 131. 
Outre qu'est ici donnée la clef du titre de la revue, des indications concernant des types nouveaux de savoirs sur les techniques apparaissent. Des projets de connaissance originaux sont en effet en plein bourgeonnement dans les années trente et quarante, et la revue S.É.T. va fournir l'occasion de leur donner la parole. On peut citer principalement la mécanologie, la cybernétique et les «sciences comparées », dont on ne peut ici qu'esquisser un bref aperçu.

La mécanologie est un projet de science, exposé dans l'ouvrage célèbre écrit en 1932 parl'ingénieur et architecte Jacques Lafitte, Réflexions surlascience des machines. Considérant un règne des machines, tout comme il existe un règne végétal ou un règne animal, il s'agit d'étudier les machines « pour elles-mêmes, en tant que phénomènes, et non plus seulement pour les phénomènes dont elles sont le siège $\|^{15}$. Les sciences du vivant servent de modèle, pour classer, décrire et expliquer les différences entre les machines, et leur évolution. La mécanologie repose ainsi sur une "mécanographie ", préalable descriptif fournissant le matériau indispensable à la démarche explicative. Ducassé et Lafitte se connaissent : l'année suivant la parution de ses Réflexions, ce dernier écrit un petit article de présentation dans la Revue de synthèse à laquelle collabore le premier ${ }^{16}$. Lafitte ne publiera cependant qu'un seul article dans S.É.T. (le n³1-32 de 1952), ce qui est assez peu au regard, d'une part, des références régulières et élogieuses par lesquelles le premier mentionne le second, et, d'autre part, de l'importance croissante que vont prendre dans la revue la cybernétique et les sciences comparées.

La cybernétique peut être définie de différentes façons. À l'origine, il s'agit d'un schématisme issu d'analogies entre servomécanismes et organismes ; ce qui était alors sous sa forme la plus élaborée une catégorie de modèles a parfois été extrapolé pour désigner tous les modèles de machines, voire la " méthode des modèles " en général ${ }^{17}$. En France, le polytechnicien Robert Vallée fonde le "Cercle d'Études Cybernétiques ॥ en 1949 18. L'un de ses

15 Jacques Lafitte, Réflexions sur la science des machines, Paris, 1932, Bloud \& Gay, p. 17.

16 Jacques Lafitte, "Sur la science des machines», Revue de synthèse, T. VI, n², oct. 1933, pp. 143-158.

17 Ronan Le Roux, La cybernétique en France (1948-1970). Contribution à l'étude de la circulation interdisciplinaire des modèles et des instruments conceptuels et cognitifs, thèse de doctorat de l'EHESS, 2010 ; Ronan Le Roux, " L'impossible constitution d'une théorie générale des machines 1 , Revue de synthèse, vol. 130, nº 1, 5-36, 2009, pp. 5-36.

18 Vallée, alors ingénieur en ballistique, fut enthousiasmé par la lecture du classique de Norbert Wiener, Cybernetics. Le camarades de l'X, Jean Sousselier, qui connaît le frère de Ducassé (l'économiste Edouard Ducassé), va devenir président de l'Association pour l'Étude des Techniques. Des liens étroits se tissent donc, et S.É.T. va quasiment devenir le bulletin officiel du CÉCyb : celui-ci se voit attribuer une rubrique régulière, tandis que Vallée prend en charge l'essentiel de la bibliographie. L'affinité quasi-immédiate de S.É.T. avec la cybernétique, sensible dès le n² (décembre 1948) qui annonce la " naissance d'une science nouvelle " quelques semaines après la sortie du livre Cybernetics du mathématicien Norbert Wiener ${ }^{19}$, culmine avec un numéro spécial (n³5-36, 1953-54) publiant une série de conférences données à la Maison des Sciences en mars 1953, par le physicien Louis de Broglie, le physiologiste Alfred Fessard (du Collège de France), le mathématicien Georges Théodule Guilbaud, l'ingénieur Julien Lœb, et le pionnier français de l'informatique, Lovis Couffignal. Tous (hormis Lœb), Ducassé et Lafitte compris, sont membres du CÉCyb, dont de Broglie assume la présidence d'honneur. Les réunions se tiennent dans les locaux de l'IHST, que Ducassé met à la disposition du Cercle. Ducassé fait d'ailleurs plus qu'ouvrir les lignes de S.É.T. à la cybernétique, puisque, d'après Vallée, c'est par son intermédiaire qu'un numéro entier de Thalès lui est consacré.

Couffignal, qui s'est aussi intéressé rapidement à la cybernétique, projetait néanmoins de faire valoir en priorité des idées personnelles qu'il développait dès les années trente. II propose de remplacer le terme " cybernétique » par celui de «sciences comparées ». L'idée essentielle qui demeure à travers les différents noms des projets doctrinaux de Couffignal (" analyse mécanique ", " mécanique comparée ", " sciences comparées ॥), c'est la mise au point d'une méthode d'analyse capable de déterminer le domaine du mécanisable en général - "acquérir une vue d'ensemble des diverses activités où l'homme a été ou pourra être remplacé par la machine [et] établir des lois de substitutions " - sans limitation disciplinaire a priori. Une telle démarche ne peut être à proprement parler une science, dans la mesure où elle ne repose pas sur des raisonnements hypothéticodéductifs. C'est un usage rationnel du raisonnement analogique qui doit permettre une connaissance

CÉCyb a rassemblé une quarantaine de mathématiciens (Guilbaud, Le Lionnais, Mandelbrot, Riguet), d'historiens des techniques (Russo, Gimpel), d'ingénieurs (Vallée, Pèlegrin), médecins, philosophes, etc., entre 1949 et 1953 ; cf. notre thèse La cybernétique en France (1948-1970), ch. 212.

19 Norbert Wiener, Cybernetics or control and communication in the animal and the machine, Paris, Hermann, 1948. 
des machines abstraites, pour laquelle Couffignal établit une filiation jusqu'au "Cours de machines " que Monge donnait à l'École Polytechnique. Mais la Société Française de Sciences Comparées ne tiendra que deux réunions, et Couffignal, pour des raisons restant à déterminer, préférera se rattacher au label cybernétique - à condition d'y critiquer Wiener et de l'accommoder à ses idées. C'est ainsi qu'il donne une définition célèbre de la cybernétique : «art de rendre efficace l'action », qui participe au demeurant d'une modalité générale de I'appropriation française de la cybernétique : celle-ci est rapprochée d'autres courants, comme la théorie des jeux, la recherche opérationnelle ou encore la "praxéologie ", qui ont pour optique de rechercher des optimums de rendement ou de décision.

On aperçoit, entre les aspirations de Ducassé et les savoirs qu'il accueille dans sa revue, des points de convergence essentiels qui dépassent une simple compatibilité fortuite. La vocation interdisciplinaire de la mécanologie comme de la cybernétique s'inscrivent à rebours de la division du travail chère aux positivistes. Là où Comte considère que l'organisation de cette division ne ferait que tirer rationnellement les conséquences d'une coupure naturelle (puisque " la haute capacité dans les sciences théoriques et la haute capacité dans les sciences d'application sont essentiellement distinctes et à tel point qu'elles s'excluent mutuellement, qu'elles ne sauraient exister dans la même tête " ${ }^{20}$ ), Lafitte écrivait dans son article de 1933 qu'« il est peu d'objets, dont l'entière connaissance suppose, à un tel point, l'application simultanée de toutes les disciplines scientifiques. (...) la connaissance des techniques, et partant des machines, suppose une synthèse intégrale du savoir et des facultés humaines. (...) son retard à se former tient à une déficience primitive de l'esprit de synthèse $1{ }^{21}$. On peut soupçonner que Lafitte est à l'origine des doutes ayant saisi Ducassé au sujet de la capacité du positivisme à rendre compte de la réalité technique.

Wiener, contribuant aussi bien à la modélisation du mouvement brownien qu'à la conception de prothèses sensori-motrices, à l'analyse harmonique qu'au guidage des fusées, s'élève notoirement contre la parcellisation bureaucratique de la recherche scientifique et technique dans les lignes qui ouvrent son livre Cybernetics. C'est ce que Ducassé met au crédit de la cybernétique, dans un petit article écrit avec Robert Vallée en 1958 :

20 Auguste Comte, cité par P. Ducassé, Essai sur les origines intuitives du positivisme, Appendice I A, p. 230.

21 Jacques. Lafitte, "Sur la science des machines ", Revue de synthèse, $\mathrm{t}$. $\mathrm{VI}, \mathrm{n}^{\circ} 2$, oct. 1933, p. 145.
"Le don de " joyeux avènement " de la cybernétique, c'était d'apporter, avec des exemples précis de raccordement entre domaines scientifiques autrefois isolés, l'idée directrice de tous les problèmes analogues et l'ébauche des méthodes propres à les résoudre ${ }^{22}$ \%).

Triple intérêt, donc, de la cybernétique aux yeux de Ducassé : comme réaction à la fragmentation toujours plus accentuée des disciplines scientifiques, comme collaboration étroite et fructueuse entre théorie et application selon des modalités non positivistes, et comme opportunité que, vue comme science de l'action de par sa réception française, la cybernétique s'inscrive à la place de la "science concrète " abandonnée par Comte. Ce dernier méprisait l'enseignement de "science des machines" professé à l'école Polytechnique ${ }^{23}$, qui est exactement l'héritage que Couffignal essaye alors de réactiver. Ce point n'échappe pas à Ducassé qui, dans son article " Auguste Comte et la philosophie des techniques », fait le rapprochement entre les « sciences comparées » de Couffignal et la «science concrète » de Comte.

Les modalités historiques et conceptuelles de couplage de ces trois projets théoriques (mécanologie, cybernétique et sciences comparées), dans les années quarante et cinquante, appellent une analyse qui dépasse de loin le cadre de cet article ${ }^{24}$; la revue S.É.T. apparaît un moment comme un lieu privilégié de ces échanges, une étape entre des traditions aux trajectoires diverses et des élaborations aux destins variables. Avec la revue, il s'agit de donner une existence sociale aux idées, de faire consister un lieu d'échange là où Comte instaurait une séparation de l'ingénieur, du philosophe et du savant.

Les grandes lignes éditoriales de S.É.T. figurent dans un cartouche, d'abord en deuxième de couverture, puis, à partir de 1953, en quatrième de couverture ; l'évolution de ce cartouche suit pas à pas l'évolution des centres d'intérêt de Ducassé.

Les " confrontations entre techniques et valeurs" sont certes plutôt courantes, à cette époque où l'on débat beaucoup sur l'humanisme et la technocratie, mais on pourra noter que le thème des valeurs

22 Pierre Ducassé, Robert Vallée, « Grandeur, décadence et ténacité des thèmes cybernétiques ॥, S.É.T. n 59-60, 1958. 23 François Vatin, "Comte et Cournot. Une mise en regard biographique et épistémologique ", Revue d'Histoire des Sciences Humaines n8, pp. 9-40, 2003.

24 Ronan Le Roux, "L'impossible constitution d'une théorie générale des machines ? ॥, op. cit. Le contenu de S.É.T., bien sûr, ne se limite pas à ces trois courants, comme on en jugera facilement en consultant le sommaire en annexe du présent article. 
A NOS LECTEURS ET CORRESPONDANTS

S.E.T., Revue de documentation, présente périodiquemen un BILAN des Informations scientifiques et techniques: - desESQUISSES de syntheses rationnelles:
- des CONFRONTATIIONS entre techniques et valeur:

\section{- Informations Scientifiques \\ Esquisses de Synthèses rationnelles Confrontations entre Techniques et Valeurs Travaux du Cercle d'Etudes Cybernétiques}

1952

apparaît ici en vertu d'un intérêt philosophique général de Ducassé, comme il ressort à la lecture de son vade mecum de 1941 sur Les grandes philosophies : c'est cette notion de valeur qui fait le pivot de la dernière partie sur la philosophie contemporaine (philosophie qui, d'ailleurs - ainsi s'achève l'ouvrage - « compenserait les effets du machinisme »).

Les "esquisses de synthèses rationnelles " sont probablement à mettre en rapport avec le Centre de Synthèse d'Henri Berr, que Ducassé connaissait bien. II collabore à la Revue de Synthèse dès octobre 1932. On peut supposer qu'il a tenu à reproduire avec S.É.T. quelque chose du dispositif intellectuel de la Revue de Synthèse, lui-même inspiré de L'année sociologique, à savoir la tenue aussi systématique que possible de compte rendus de lecture, auxquels s'ajoutent des annonces de conférences et de parutions dans la rubrique "Informations". Ce qui se profile derrière cette démarche, c'est une coordination des savoirs, pour que les rapports qui les lient ne soient pas perdus de vue dans un contexte d'éparpillement (rappelons que le CNRS ne trouve son véritable essor qu'après la guerre); S.É.T. serait ainsi aux techniques une réplique miniature de ce que la Revue de Synthèse est aux sciences. Le texte fondateur de l'Association SÉT, qui ouvre le premier numéro de la revue, insiste précisément sur la vocation de " coordination », ainsi que d'u assimilation historique et philosophique ", de la revue. On aperçoit, à l'occasion de ce rapprochement, l'intérêt qu'ont pu éprouver des membres du Centre de Synthèse à l'égard de la visée interdisciplinaire de la cybernétique ${ }^{25}$.

Le Cercle d'Études Cybernétiques n'est mis en vedette dans le cartouche que l'année à la fin de laquelle il cesse son activité (1953). Le CÉCyb ne sera pourtant jamais officiellement dissout. La référence du cartouche restera jusqu'en 1957, où elle sera

25 Parmi les membres du CÉCyb, outre F. Le Lionnais, figure une autre collaboratrice du Centre de Synthèse : Suzanne Colnort, assistante de Maurice Daumas, qui publie en 1955 un article sur la cybernétique dans la Revue de Synthèse.
1953

1960

remplacée simplement par « Cybernétique (Travaux et bibliographie) $).$

La mention «Recherche de Sciences Comparées» est incluse à partir du n³9-40 de 1954, sans doute pas par simple intérêt intellectuel. Cela fait en effet deux ans que les Sciences comparées existent théoriquement, sans s'être vues attribuer de rubrique particulière dans S.É.T., tandis que dans la pratique les réunions n'auront lieu qu'en 1956. Pourquoi la date de 1954 ? Entre temps, Ducassé et Couffignal se sont rapprochés, et se soutiennent mutuellement : S.É.T. accorde une part importante de ses parutions à Couffignal et aux sciences comparées (ce qui apparaît nettement à partir du n³9-40), comme en témoignent également les articles de méthodologie générale d'un chimiste du CNRS, Paul Renaud, que Couffignal a fait intervenir dans sa Société de Sciences comparées ; réciproquement, Couffignal ouvre très vraisemblablement ses réseaux à Ducassé, puisqu'on retrouve ce dernier, à partir de 1953, membre du jury du Certificat d'aptitude au Professorat dans les Écoles Nationales professionnelles et Collèges techniques, présidé par Couffignal, et, surtout, celui-ci va jouer un rôle probable dans le transfert de Ducassé au Cnam, entre 1956 et 1960, puisqu'en tant qu'inspecteur général de l'enseignement technique, il côtoie très régulièrement Louis Ragey, directeur du Cnam depuis 1940, et lui aussi inspecteur général de l'enseignement technique depuis 1939, ayant conservé ses fonctions et assistant à toutes les réunions des inspecteurs aux côtés de Couffignal.

Ainsi, la dernière mention du cartouche, «Méthodes d'expression de la pensée scientifique et technique ", qui apparaît en 1960 avec le n69-70, correspond mot à mot à l'intitulé de la chaire que va occuper Ducassé au Cnam dans les années soixante. Cette chaire est créée spécialement pour lui, après qu'il ait occupé deux ans un poste de chargé d'enseignement à la suite de son départ de l'IHST en 1955. Pour introduire son enseignement, en 1956, il reçoit l'appui ferme de Max Serruys ${ }^{26}$, professeur au Cnam (et dans d'autres

26 Rapport de séance du 4 juin 1956 du Conseil de perfectionnement du Cnam, pp. 3-4. Lors de la discussion qui suit l'au- 
grandes écoles) et spécialiste éminent des moteurs à combustion, qui écrira aussi la préface de Les techniques et le philosophe. La transformation de son cours en chaire semble faire l'unanimité au Cnam, bénéficiant d'un autre appui de longue date, celui du physicien Louis de Broglie, qui le connaît depuis la création du CECyb dont il fut le parrain. Ducassé, dès lors professeur au Cnam, abandonne son poste de Besançon et les déplacements fatigants qui en découlaient. II publie, en rapport avec son cours sur les méthodes d'expression, un ouvrage en deux tomes chez Dunod ${ }^{27}$. Cet intérêt pour «l'art de s'exprimer », comme il titre l'un de ses articles (S.É.T. n77-78), reste en fait en continuité avec son investissement dans l'enseignement technique et ses réflexions sur l'éducation des techniciens (peut-être, aussi, avec les activités de Couffignal, qui crée les Brevets de Technicien Supérieur, et cherche des applications de la cybernétique à la pédagogie), à une époque où l'on nourrit des espoirs de reconnaissance de la dignité de l'enseignement technique. Ducassé crée en 1962, avec ses étudiants du Cnam, les « Cercles SÉT »; ces " cercles d'étude et d'entraînement dans le domaine des méthodes d'expression " ne vont cependant pas perdurer.

Les intérêts de Ducassé se diversifient, ce qui se traduit peut-être par une dispersion de ses engagements et une raréfaction des numéros de S.É.T. : dans les années soixante, en sus des méthodes d'expression, il s'investit en effet dans des travaux portant sur l'économie et ce qu'on n'appelle alors pas encore le management. II collabore avec l'économiste François Perroux, qui lui commande un article pour le tome IX de L'Encyclopédie Française qu'il coordonne («L'univers économique et social»), et qui lui confie une collection de ses Cahiers de l'Institut des Sciences Economiques Appliquées, baptisée, on ne s'en étonnera pas, "Évolution des techniques et progrès de l'économie $12^{28}$. Rappelons que le frère de Ducassé est économiste. Par ailleurs, il collabore à des travaux portant sur la gestion des administrations ${ }^{29}$.

dition de Ducassé, Serruys indique avoir siégé dans le même jury que lui à l'École Normale d'Enseignement technique.

27 Formation intellectuelle et méthodes d'expression, t. 1 (1962), t. 2 (1967).

28 II s'agit de la série AD. En fait seulement deux numéros seront publiés, en mars 1961 et février 1963.

29 Manuel du chef administratif, avec Cl. Vautrin et J.-P. Jolly, Entreprise Moderne d'Édition, 1966 ; et Analyse et contrôle de gestion d'un organisme administratif : L'expérience de la Caisse nationale de retraite des ouvriers du bâtiment et des travaux publics C.N.R.O., avec l'assistance de H. Girard et J. Richard, Dunod, 1968.
Cette diversification, et le départ du milieu philosophique universitaire, signe sans doute une forme de rupture que la continuité manifestée dans l'intérêt pour l'enseignement technique ne doit pas masquer : dépit, sans doute, d'avoir été écarté in extremis de la direction de I'IHST au profit de Canguilhem. On a là sans doute une raison expliquant qu'un ouvrage en projet, Auguste Comte et la philosophie des techniques, ne fut jamais achevé.

Nous savons peu de choses de l'espace de réception de S.É.T. La revue était distribuée par les Services d'Édition et de Vente des Publications de l'Éducation Nationale ${ }^{30}$, dont les fichiers de vente n'ont pas été conservés pour cette période; hormis la diffusion de la revue dans le réseau du Centre National de Documentation Pédagogique, on ne sait donc pas grand chose de son public.

Pour ce qui relève du réseau plus informel de Ducassé, il apparaît que S.É.T. s'avère un vecteur important de constitution du Cercle d'Etudes Cybernétiques, puisqu'un certain nombre de membres entendent parler du CECyb en lisant S.É.T., et contactent la revue pour se renseigner ou carrément demander à s'inscrire au CECyb. La revue sert aussi à connecter le CECyb à l'Etranger: on trouve une lettre d'un américain qui cherche à constituer une bibliographie sur la cybernétique française, et à la comparer à son homologue américaine ; plus encore, S.É.T. met en relation le CECyb avec le Centro Italiano de Cibernetica ${ }^{31}$.

L'interprétation de cette mise en réseau appelle des remarques importantes. Si le rôle de S.É.T. dans cette connexion des groupements de cybernétique est susceptible de représenter la portée encyclopédique de la technique, en revanche on peut douter qu'il l'incarne. En effet, sous sa forme la plus opératoire, la cybernétique a consisté en la mise en circulation de modèles de machines en sciences biologiques et humaines, autrement dit en la répercussion d'un enrichissement de la pensée mécaniste dans différents domaines du savoir ; et c'est précisément ce que n'a pas été la revue S.É.T. : un catalogue ou un lieu de développement de modèles; et cela sans doute notamment parce que le CECyb lui-même n'avait pas abouti à définir

30 Ancien nom du Service Culture Éditions Ressources pour I'Éducation Nationale, toujours installé au 13 rue du Four (Paris $6^{\mathrm{e}}$ ), dans le même immeuble que I'IHPST.

31 Correspondance personnelle de Robert Vallée ; nous en reproduisons une partie en annexe de notre thèse. L'un des membres du C.I.C., l'ingénieur Delfino Insolera, est aussi membre du CECyb ; on trouve notamment au C.I.C. le célèbre statisticien Bruno de Finetti. 
ainsi sa raison d'être, mais cherchait celle-ci au gré des différents horizons d'attente et des différentes modalités de réception et d'appropriation de la cybernétique qui furent ceux des acteurs français. Alors que les périodiques techniques jouent en général un rôle important dans la structuration de groupes et d'identités professionnelles ${ }^{32}$, S.É.T. ne rentrait justement pas dans ce cadre : revue sur les techniques elle n'a pas été une revue technique, tandis que les cybernéticiens ne constituaient pas un véritable groupe avec une identité définie particulièrement dans le contexte qui est celui de la France d'après Guerre, défavorable au développement de telles pratiques de modélisation interdisciplinaire ${ }^{33}$.

Peut-être l'originalité de l'entreprise éditoriale de Ducassé, sa curiosité à l'égard des initiatives qu'il accueille, impliquait-elle un lectorat quelque peu hasardeux. II semblerait toutefois que l'incertitude sur les débouchés ne freinait pas l'enthousiasme éditorial de Ducassé ; tandis que sa femme sténographiait et ronéotypait les premiers numéros, lui-même, par ce que l'on peut s'amuser à interpréter comme une sorte de réflexivité inversée, suivait de près les procédés de fabrication à l'imprimerie. L'incertitude éditoriale est sans doute le prix d'une démarche "d'avant-garde ", indispensable selon Ducassé à une intelligence contemporaine des techniques. L'étude historique en elle-même, bien qu'importante (on aura remarqué que l'Histoire des techniques de Ducassé précède nettement les grands travaux de référence de Daumas, Gille ou encore Haudricourt), ne suffit pas à saisir une réalité au rythme très particulier, dont l'accélération défie la temporalité contraire de la méditation philosophique :

" La technique pose présentement au philosophe, en quête de documentation significative, un problème beaucoup plus brutal et beaucoup plus caractéristique par sa vitesse de développement. Celle-ci, en effet, met en cause directement le sens des manifestations techniques, parce qu'elle voile certaines coordinations, parce qu'elle en impose parfois de nouvelles, et surtout parce qu'elle les transfigure en général complètement dans leur ensemble. Un phénomène dont l'intelligence avait péniblement pris possession, sous une forme déjà historique, même si elle est très

32 Patrice Bret, Konstantinos Chatzis \& Liliane Pérez, La presse et les périodiques techniques en Europe, 1750-1950, Paris, L'Harmattan, 2008.

33 R. Le Roux, La cybernétique en France (1948-1970, op. cit.. proche de nous, se trouve parfois complètement périmé, quand il est - enfin - bien compris. [en note :] En ce sens l'information du philosophe des techniques, contrairement à toute méthode saine et traditionnelle d'érudition doit être d'avant-garde et parfois par sources très inégales (sources de publicité - sources de documentation indirecte et insuffisamment contrôlée sources d'anticipation des spécialistes d'une technique donnée) ${ }^{34}$ ».

La recherche de nouveauté se remarque aussi par la variété des approches des divers spécialistes qui tentent de cerner le fait technique dans leur domaine, ainsi qu'on en juge rapidement en consultant le sommaire des numéros (voir en annexe). Cette pluralité des démarches, mise en perspective avec le constat de l'insuffisance de la philosophie, s'avère ainsi corrélative de la réalité et de la consistance irréductibles de l'objet de la revue - le phénomène technique - , faisant d'autant mieux saillir cette autonomie qui n'était pas suffisamment prise en compte aux yeux de Ducassé.

Laphilosophienes'en trouve paspourautantchassée. Ducassé lui-même reste avant tout philosophe. On reconnaît une posture d'inspiration phénoménologique, lorsque la démarche philosophique est présentée comme quête de sens (à l'égard d'un "objet " ou phénomène qui, justement, est ou entraîne une perte de sens ${ }^{35}$ ). Ducassé, par contre, ne semble pas avoir pris connaissance du texte de Heidegger, « La question de la technique ». II est vrai que la traduction française de ce texte était contemporaine de la rédaction de Les techniques et le philosophes, puisque les deux paraissent la même année. Si l'utilisation du mot "péril " dans les deux textes est donc une pure coïncidence, en revanche, le rapprochement entre les deux penseurs est instructif à l'égard de leur visée. Ce que tous deux ont en vue, c'est l'essence de la technique, même si Ducassé ne le formule pas tel quel; ce qui est " dévoilement " chez Heidegger s'appelle chez lui "vision directe ». Mais alors que Heidegger en appelle à une "méditation " qui, pour des raisons intrinsèques, nécessiterait d'être elle-même dépourvue de tout élément de technicité, Ducassé, en revanche, semble considérer que la Technique,

34 P. Ducassé, Les techniques et le philosophe, op. cit., pp. 86-87. 35 Ibid., pp. 24, 81. On peut remarquer que l'analyse husserlienne de l'évacuation du sens dans les sciences de la nature se focalisaitjustementsur la dimension technique de la mathématisation. La technique apparaît donc à ce titre, une fois encore, constituer un thème privilégié de l'investigation philosophique. 
"porteuse de sa propre philosophie ", serait à même de fournir une intelligence réflexive spécifique. On peut donc chercher - et c'est certainement ce que fait Ducassé avec S.É.T. - un recours à une pensée technique pour penser les techniques:

"Ce qui est nouveau et ce qui fait " défi " c'est que notre intelligence doit ici changer radicalement la direction habituelle de son ingéniosité technique : inventer, si l'on peut dire, une "technique contre ses techniques" ou au moins contre leur allure menaçante d'aveuglante fatalité. D'autre part, cette innovation doit être assez prompte pour restituer à l'homme son pouvoir d'arbitrage avant que la technique l'ait, lui aussi, inclus dans le mécanisme qu'il doit juger et orienter (...) Au défi que la technique pose à l'homme par la consistance nouvelle de son tissu, par sa puissanced'enveloppementcapabled'éluder toute question, le philosophe doit répondre par une prise de conscience accrue de ce qui fut toujours son art, sa mission caractéristique : savoir mettre "techniquement » en question ce qui tend à soustraire I'homme à sa dignité de « questionneur $\|^{36} »$.

D'autre part, la philosophie de Ducassé révèle une orientation bergsonienne, sensible à travers la thématisation des rapports entre temps et technique. Passionné d'horlogerie, Ducassé s'intéresse de près aux techniques de mesure du temps. Sa nomination, parallèlement à ses activités à l'IHST, à la Faculté des lettres de Besançon - ville qui accueille une véritable "technopole " de l'horlogerie —, au début des années quarante, est une heureuse coïncidence qui va lui permettre de nover des liens avec l'industrie horlogère; ainsi s'explique la profusion des "réclames" ornant les trente premiers numéros de S.É.T., mais aussi le fait qu'il coordonne un numéro spécial de la revue La Suisse horlogère sur la cybernétique ${ }^{37}$. Avant S.É.T., Ducassé s'investit dans la revue Thalès de I'IHST, dont il est I'un des trois membres du comité de rédaction ${ }^{38}$. Dans le tome $V$

36 P. Ducassé, Les techniques et le philosophe, op. cit., pp. 14, 16. 37 Numéro de mars 1957. On y trouve l'article de Ducassé \& Vallée déjà cité (réédité dans S.É.T.), ainsi qu'un article de R. Dessoulavy : "Limites à la précision de mesure d'un intervalle de temps $॥$.

38 On peut croire qu'il retire de cette expérience quelque chose pour S.É.T., ne serait-ce qu'un point commun avec la Revue de Synthèse: Thalès, qui présente des travaux historiques et philosophiques de l'IHST, se veut d'après Abel Rey de Thalès, outre l'article « Pour l'histoire de la mesure du temps », c'est encore une fois un petit article, "Le "temps» des techniques ", qui effectue les liaisons conceptuelles fondamentales. La technique n'est pas de la science appliquée, donc le temps de la technique n'est pas le temps des sciences:

"Même si la technique était de la science appliquée, l'application supposerait encore l'implication d'une durée : celle de l'homme qui imagine et réalise ou celle du groupe qui institue l'application ${ }^{39} \mathrm{ll}$.

De par sa « spontanéité " et "la ténacité de son inscription dans le temps », le fait technique se déploie dans une durée vécue par l'homme :

" Le temps vécu par l'homme, quand il se mesurait seulement par l'ombre du soleil, avait certainement une autre saveur que le temps, chichement compté si l'on veut, en tous cas précisément découpé d'un citadin moderne. Mais il est tout à fait gratuit d'en inférer davantage. Il est seulement avéré que notre vie n'est pas indifférente à ce découpage aigu, de plus en plus aigu, qu' opèrent, dans sa trame, les techniques de mesure du temps, les manières d'enrichir ou d'user le temps; il y a donc lieu de se pencher sur les rapports qui en découlent. Le besoin d'une psychologie, d'une sociologie de ces techniques de la vie quotidienne [en note: à quoi se rapporte directement un livre en préparation de G. Friedmann] peut compter parmi les manifestations positives de notre participation au temps des techniques ${ }^{40} \mathrm{~m}$.

La liaison se fait ainsi entre le temps, les techniques et un troisième thème (bergsonien ?) cher à Ducassé : le loisir, auquel il consacre plusieurs articles, chapitres, et un numéro spécial de S.É.T. avec le sociologue J. Dumazedier et le philosophe B. Guillemain.

Mais ce qui apparaît aussi au détour de cet article de 1948, ce sont les points de contact que présente la philosophie de Ducassé avec des thèmes directeurs de la philosophie française des techniques contemporaine. II est vrai que, contrairement à aujourd'hui, Ducassé, suivant le discours d'un certain nombre de disciplines de l'époque, considère comme "négligeables " les techniques de l'esprit,

« un remède précieux, l'antidote même des dangers de l'esprit de spécialisation » (Thalès $n^{\circ} 1$, « Avant-Propos », p. XIX, 1933). 39 « Le « temps » des techniques », Thalès, tome V, 1948, p. 23. 40 lbid., p. 23. 
les prothèses mentales. Par contre, tout autant que le conditionnement du vécu perceptif par l'évolution des techniques temporelles, résonnera familièrement à l'esprit d'un lecteur actuel l'idée que la philosophie doit rendre compte des rapports entre " accélération » et « incertitude » :

"Quand les prétendues techniques n'auraient entre elles que ce trait commun d'imposer, ou de paraître imposer à notre rythme vital un même type d'accélération, un même décalage entre les conceptions acquises et les adaptations requises, le philosophe serait tenu provisoirement d'en faire état, c'està-dire de respecter dans cette nébuleuse de significations, le sens d'une incertitude caractéristique de l'homme ${ }^{41}$ ॥.

\section{Conclusion}

Contrairement à une optique positiviste par laquelle c'est la théorie qui aménage l'application, l'action précède ici l'inscription symbolique : le lancement de la revue S.É.T. se fait dix ans avant l'envol de l'oiseau de Minerve, peut-être par cette " avance de phase " que Ducassé attribue à la spontanéité de la pratique. Mais l'initiative éditoriale n'éclot pas isolément, sans quelques idées ou intuitions préalables; celles-ci requièrent simplement le temps de leur élaboration, qui ne se fait pas sans la revue.

La recherche voulue d'une « technique contre les techniques ॥ n'a pas franchi le pas consistant à faire de la revue elle-même un dispositif engagé de façon opératoire dans une activité positive, catalogue ou ressource pour des pratiques instrumentales interdisciplinaires (métrologie, modélisation...). Dépassant pourtant le mode de la seule réflexion philosophique, S.É.T. s'est ainsi trouvée dans un entredeux, un « non lieu » académique et institutionnel, un no man's land ne correspondant à aucune identité professionnelle définie.

Objet éditorial atypique, non tant par ses modèles d'inspiration que par ses thèmes, ses objectifs et le contexte de sa mise en œuvre, S.É.T. tire sa raison d'être de ce caractère improbable : faire exister temporairement un lieu pour permettre des échanges non assurés par l'institution et la tradition, initiative toujours précaire et sans cesse à renouveler.

41 P. Ducassé, Les techniques et le philosophe, op. cit., p. 43. 


\section{S. E. T. \\ STRUCTURE ET EVOLUTION DES TECHNIQUES REVUE DE DOCUMENTATION \\ DE L'ASSOCIATION POUR L'ETUDE DES TECHNIQUES \\ 2, Rue Mabillon - PARIS (6') \\ SOMMAIRE DES 30 PREMIERS NUMÉROS PARUS \\ (Années 1948 à 1951)}

$1^{\text {tr }}$ numéro. - 15 Novembre 1948 - Georges MATORÉ, professeur à la Faculté des Lettres de Besançon, La méthode en lexicologie, un exemple: Le Vocabulaire technique. - Centre d'Etudes philosophiques et techniques du théâtre. \& Rapports du lieu théâtral avec la dramaturgie présente et à venir. " (Rapports préalables a la semaine d'études du Centre d'études philosophiques et techniques du théatre, semaine qui a eu lieu en Sorbonne en décembre 1948, et à laquelle participèrent, entre autres, MM. Raymond BAYER, Gaston BATY, André BOLL, J. CO. PEAU, Ch. DULLIN, Henri GOUHER, Louis JOUVET, Charles LALO, LE CORBUSIER, Auguste PERRET, Etienne SOURIAU, Pierre SONREL (Directeur du Centre: André VILLIERS). - Les Bibliographies de l'avenir: Technique de la pré-annonce.

$N^{\circ} 2$. - Décembre 1948 - René BAILLAUD, Directeur de l'Observatoire National de Besancon: \& Neptune » et Le Verrier. - R. de BENGY-PUYVALLÉE: La définition de la grandeur Temps en physique théorique.

INFORMATIONS: Prix Eddington. - Archéocivilisation, - Naissance d'une science nouvelle: La Cybernetique.

$N^{\circ} 3$, - Janvier 1949 - René BAILLAUD, Directeur de l'Observatoire National de Besançon: "Neptune " et Le Verrier (fin). - Pierre MESNAGE, Maître de Conférences à la Facultè des Sciences de Besancon: Horloges à Haute et Basse Fréquence: Evolution de la métrologie du temps.

INFORMATIONS: Conférences d'Histoire et philosophie des techniques. - Congrès annonces. - Chronique de l'energie atomique.

$N^{*} 4$, - Février 1949 - D André CHARLIN, médecin spécialiste, chef du service de neuro-psychiatrie du Centre Régional Hospitalier, chargé de cours à l'Ecole de Médecine de Besançon: Intérêt technique de l'Exploration narcoanalytique (Le problème du « sérum de vérité »). - Edouard DUCASSÉ: Introduction des facteurs dynamiques dans la théorie économique.

INFORMATIONS: Exposition du Conservatoire National des Arts et Métiers: Les Chef́ d'ouuvre de l'Horlogerie. - Conírences données A l'occasion de l'Exposition, - L'horloge dite \& Atomique ».

$N^{\circ}$ 5. - Mars 1949 - Georges MATORE, Professeur à la Faculté des Lettres de Besançon: Un problème de délimitation et d'influences: la lexicologie et les sciences voisines. - Pierre DUCASSÉ, Professeur à la Fáculté des Lettres de Besancon: Servitude et Libération de l'acteur. - Charles BRIED, Professeur aux Ecoles normales de Besançon: Les "Techniques du Yoga 》 selon Mircea Eliade.

INFORMATIONS: Conférences - Union des Associations Interniationsles - Le Mètre lumineux.

$N^{\circ}$ 6. - Avril 1949 - Georges LUTFALLA, Administrateur général de l'Ecole Nationale d'Organisation Economique et Sociale, Membre du Conseil Economique: Les Institutions comptables en France et à l'Etranger, - Maurice DAUMAS: La naissance et le développement de la chimie en Chine.

INFORMATIONS: Conférences - Assemblée Société Chronométrique de Franre - 150e anniversaire de la naissance de Balzac que de Franre Thales.

$N^{\circ} 7$ - Mai 1949 - Georges LUTFALLA, Administrateur général de l'Ecole Nationale d'Organisation Economique et Sociale, Membre du Conseil Econo- mique: Les Techniques du Comptable (suite et fin). T. KAHAN, Chef du Laboratoire de Recherches pour Microondes, et C. MAGNAN, Sous-Directeur du Laboratoire de Physique Atomique du Collège de France: INFORMATIONS: - Conférences - XVe Semaine de Synthèse IV. Congrès des Sociétés de Philosophie de Langue française (Neucâtel, 13-16 septembre 1949).

$N^{\circ} 8$ - Juin 1949 - Louis de BROGLIE, Secrétaire perpétuel de l'Académie des Sciences: "L'énergie atomique et ses applications 》(Préface). - T. KAHAN, Chef du Laboratoire de Recherches pour Microondes, et C. MAGNAN, Sous-Directeur du Laboratoire de Physique atomique et moléculaire du Collège de France: « Applications industrielles des isotopes radioactifs \%. - André VILLIERS, Directeur du Centre d'Etudes philosophiques et techniques du theâtre; "Nécessité d'une tradition d'études philosophiques et techniques du théâtre ».

INFORMATIONS: Congrès International de Philosophie des Sciences (17-22 octobre 1959) - Société de Psychologie - Correspondance de Lavoisier: Appel aux érudits et aux collectionnenrs - Congres rloge gynocyclique.

$N^{\circ} 9$ - Juillet_Août 1949 - Jacques DUMONTIER, Professeur à l'Ecole d'Application de l'I.N.S.E.E., à l'Ecole Nat. d'Organisation Economique et Sociale et au Collège libre des sciences sociales et économiques: " Equilibre physique, Equilibre biologique, Equilibre économique.

INFORMATIONS: Congrès annoncés - Renouyeaux techniques en France - Reouverture et aménagement du Musee de Cluny - Festival international de Musique de Besançon -..' Disparition de «. Bookiorecast is

$N^{\circ} 10$ - Septembre-Octobre 1949 - Lucien FEBVRE, Professeur au Collège de France, Directeur d'études à l'Ecole des Hautes Etudes: "Industrialisation et Technocratie », Introduction. - Georges FRIEDMANN, Professeur au Conservatoire National des Arts et Métiers, Directeur d'études à l'Ecole des Hautes Etudes: Les technocrates et la civilisation technicienne. - Maurice BEMOL, Docteur ès-Lettres: "Valéry et la Technique $》$.

INFORMATIONS: Conférences du Palais dé la Découverte Sésnce plénière du C.N.O.F. - Congrès international de Philosophie des Sciences - Congrès international de préhistoire.

$N^{\circ} 11$ - Novembre 1949 - Jacques DUCLAUX, de l'Académie des Sciences: La macromolécule à l'état solide. Madeleine DAVID, Professeur l'Institut Français de Praque: L'architecte et le Scribe dans l'Antiquité Mésopotamienne, A propos de fouilles récentes.

INFORMATIONS: Société irançaise de Philosophie - Palais de ta Découverte - Congrès International d'histoire des Sciences - Jour nées d'information de Strasbourg.

$N^{\circ} 12$ - Décembre 1949 - M. RODANET, Président de la Sociẻté Jeager: Les Méthodes de fabrication de l'horlogerie moderne. - Edouard DUCASSÉ: L'Economie humaine dans la structure de l'Univers.

INFORMATIONS: Palais de la Découverte - C.N.O.F. - Assemblée générale de I'Association S.E.T. (Structure et Evolution des Techniques),

$N^{\circ} 13$ - Janvier $1950-\mathrm{M}$. Pierre MESNAGE, Professeur à l'Institut de Chronométrie de Besançon: Le Congrès d'électronique et de radioélectricité (Sté des 
radio-électriciens), Paris, 16-22 janvier 1950. - $R$. de BENGY-PUYVALLEE: Les techniques et la vie, une conception organique du monde moderne. - Aurel DAVID, sonne humaine.

INFORMATIONS: Palais de la Découverte - Conservatoire natio. nal des Arts et Métiers - Société irançaise de psychologie - Cen tre d'Etudes sociologiques $\rightarrow$ C.N.O.F, - Congrès annoncés: Journées de Chronométrie - Sociétés de philosophie de langue fran. çaise.

$N^{*} 1 /$ - Février 1950 - Pierre DUCASSÉ, Professeur à la Faculté des Lettres de Besançon, Secrétaire général de l'Institut d'Histoire des sciences et des techniques (Paris): Le Temps, la Technique et la Science. - André VILLIERS, Directeur du Centre d'Etudes Plílosophiques et techniques du Théâtre: L'Expression collective au théátre (Semaine d'études de mars 1950).

INFORMATIONS: Palais de la Decouverte - Centre International de Synthese - Comité national de 1'Organisation française - Institut d'Histoire des sciences et des techniques - Journées nationales d'Etudes pédagogiques des proiesseurs de philosophie.

$N^{n} 15$ et 16 - Mars.Avril 1950 - Max SORRE, Profesceur à la Sorbonne Les techniques de production et de transformation des matières premières (conclusions). Ernest KAHANE, Directeur de Laboratoire au C. N. R. S.: La Synthèse organique industrielle.

INFORMATIONS: Palais de la Déconverte - Centre d'Etudes sociologiques - Congrès: Société Chronométrique de France, Lyon, mai 1950. - L'unification de l'alphabet Braille.

$N^{0 *} 17$ et 18 - Mai_Juin 1950 - Pierre DUCASSÉ, Prolesseur à la Faculté des Lettres de Besançon: La Technique et le Loisir. - Notes Bibliographiques. Techniques nouvelles: L'Energie solaire.

INFORMATIONS: Expositions: I'ouvre scientifique de Blaise Pascal et trois

$N^{\circ} 19$ et 20 - Juillet.Octobre 1950 - René BAILLAUD, Directeur de l'Observatoire National de Besançon: Orientation actuelle du Contrôle de Réglage des Montres à l'Observatoire de Besançon. - Daniel LACOMBE, Agrégé de mathématiques, Attaché de Recherches au C. N. R. S.: La Géométrie de Hilbert et l'Axiomatique actuelle.

INFORMATIONSt Exposition du Palais de la Découverte (Psydaii) - Horlogerie et Photo-électricité, - A Travers les Revues. Iniormations diverses.

$N^{\circ \kappa} 21$ et 22 - Novembre 1950 - Février 1951 - Pierre DUCASSE, Professeur à la Faculté des Lettres de Besancon, Secrétaire général de l'Institut d'Histoire des Sciences et des Techniques (Paris): La Technique et le Loisir (suite et fin). - Daniel LACOMBE, Agrégé de nathèmatiques, Attachè de Recherches au C. N. R. S $\mathrm{La}$ géométrie de Hilbert et l'axiomatique actuelle (suite et $\operatorname{fin}$ ).

INFORMATIONS: Sciences et Philosophie (Palais de la Découverte, Centre international de Synthèse, Institut d'Histoire des Sciences et des Techniques, Société française de Philosophie) Technique et organisation (Cnof - Club de l'Horlogerie) Technique théatrale: le théâtré et le problème des loisirs.

Nos 2.3 et $2 / 4$ - Mars_Avril 1951 - Louis de BROGLIE, Membre de I'Académie Française, Secrétaire perpétuel de l'Académie des Sciences: La Science au « Siècle des Lumières $»_{0}$ - André DANJON, Membre de l'Institut, Directeur de l'Observatoire de Paris: Une science nouvelle: la Radioastronomie. - Pierre DUCASSÉ, Professeur à la Faculté des Lettres de Besancon: Les méthodes de l'Expertise et la Philosophie des Scien. ces.

INFORMATIONS: Science et Technique (cercle de Cybernétique Docteur en droit: Vers une théorie juridique de la perchiatrie). - Congrès international d'Histoire des Sciences (Amster.

naire de Daguerre) - Technique et organisation: 9 Congrès inter. national de l'organisation scientifique - let Congrès du progrès scientifique et techniq̨ue) - Philosophie et culture générale A travers les Revues - Assemblée générale de S.E.T. (Structure et Evolution des Techniques).

$N^{\circ} 25$ el 26 - Mai.Juin 1951 - André DANJON, Membre de l'Institut, Directeur de l'Observatoire de $\mathrm{Pa}$ rie: Une science nouvelle: la Radioastronomie (suite et fin). - Pierre MESNAGE, Professeur à la Faculté des Sciences et à l'Institut de Chronométrie de Besançon: La Célébration du deuxième centenaire de la nais. sance d'Antide Janvier, à Paris et à Saint-Claude. - Conservatoire National des Arts et Métiers: Pendules et horloges d'Antide Janvier (1751-1835), Exposition du 12 juin 12 juillet 1951: catalogue documentaire. - Raoul HUS SON, ancien élève de l'Ecole Normale Supérieure, Docleur ès-Sciences: La phonation. - Ceorges HEY. MANN, Professeur de philosophie; La mesure en Photométrie.

INFORMATIONS: Science et Technique Cercle Cybernétique (CECYB) - Centre international de Synthese - Palais de la Dé. couverte - Deuxième centenaire de la naissance d'Antide Janvier, etc... - Technique et organisation - Culture générale et philosophie - A travers les Revues, etc..

$N^{\circ \circ} 27$ et 28 - Juillet.Novembre 1951 - U. ZELBSTEIN, Ingénieur-Docteur, Ingénieur-conseil à la S. N. E. C. M. A.: L'Homme et les hommes. - R. VALLEE, Ingénieur de l'Armement: Quelques themes initiaux de la Cybernétique, - G. HEYMANN, Professeur de Philosophie: La mesure en Photométrie (II): Le postulat de la mesure, - XXX: Aperçu économique de la Suisse.

INFORMATIONS; Science et Technique (Cercle de Cybernétique Association des Ingentieurs électroniciens - Société chronométrique
de France, ete.... Histoire et Philosophie des Sciencest MM. Bachelard, Costabel, Schuhl (coniérences) - Centre international de Syn. thèse (170 semaine de syntèse) - Palais de la Decouverte - A travers les Revues.

$N^{\circ s} 29$ et 30 - Décembre 1951 - Avril 1952 - Max SORRE, Professeur à la Sorbonne: L'Homme moderne dans l'oekoumène. - Robert VALLÉE, Ingénieur de l'Armement: La Cybernétique et la théorie de l'information. - André VILLIERS, Directeur du Centre d'Etudes philosophiques et techniques du théâtre: Théâtre et l'echnicité aux Etats-Unis. - Marius LAVET, Ingénieur A. M. et E. S. E., Ingénieur à la Société anonyme des Etablissements Léon Hatot: Au sujet de l'invention in. dustrielle (correspondance). - XXX: Aperçu économique de la Suisse (suite et $\mathrm{fn}$ ).

INFORMATIONS: Sciences et Technique: Cercle de Cybernétique (CECYB) - Association des Ingénieurs électroniciens - Pbysigue et Biologie (réunions Louis de Broglie) - Société Chronométrique de France - La Montre \& Electronic \$ - Histoire et Philosophie des Sciences - Congrés annoncés - A travers les Revues.

Chaque numéro de la Revue S. E. T. contient à la suite des articles et des informations, une très importante partie documentaire: textes d'analyses et de comptes rendus, textes d'annonce et de pré-annonce d'ouvrages français et étrangers, selon le plan suivant:

Mathématiques pures et appliquées - Cybernétique Physique et Mesure du Temps - Chimie et Biochimie Biologie - Psychologie et Pédagogie - Psychiatrie Economie et Sociologie - Technologie (généralités, mécanique et métallurgie, électrotechnique et électronique, divers) - Histoire des Sciences et des Techniques - Histoire de la Civilisation - Philosophie.

A cette documentation est jointe la liste des sommaires des principales revues scientifiques et techniques ou des revues historiques et philosophiques reçues par l'Association S. E. T.

Chaque numéro se termine par un Index des noms d'auteurs et un Index des noms d'éditeurs, soigneusement établis.

ABONNEMENTS: Un an: France: $750 \mathrm{fr}$. - Etranger : $1.000 \mathrm{fr}$

COMPTE CHẼUE POSTAL: Paris 67.1832 - S.E.T. Associntion, 2, me Mabillon, Paris $\left(6^{e}\right)$.

Certains numéros sont devenus rares - Prix du numéro spééimen: $150 \mathrm{fr}$.

Compose pai S.E.T. ASSOCIATION, 2, Rue Mabillon, PARIS 6* - Le Directeur Gérant de la publication: Ch. LECLABART. - Imprimeic Coopéraive, Dole 


\section{Sommaire des numéros postérieurs à 30 (articles seulement) :}

31-32 Mai-déc. 1952 Gaston BACHELARD : La vocation scientifique et l'âme humaine.

Franziska BAUMGARTEN-TRAMER : Intérêts commerciaux chez les ouvriers.

Louis Couffignal : L'organisation des recherches en Sciences comparées.

Jacques LAFITE : Responsabilités dans la construction; esquisse d'une méthode générale pour leur analyse ; Etude d'un cas concret.

Uri ZelbSteIn : Connaissance analytique et synthèse scientifique.

33-34 Janvier-juin 1953 André LeROI-GOURHAN : Origine et diffusion de la connaissance scientifique.

35-36 Juillet 53 - Jan. 54 « La Cybernétique ».

Louis Couffignal: Méthodes et limites de la cybernétique.

Julien LØєB: Information, communications et servomécanismes.

Alfred FESSARD : Points de contact entre neurophysiologie et cybernétique.

Georges Théodule Guilbaud : Pilotes, stratèges et joveurs.

Lovis de Broglie: Sens philosophique et portée pratique de la cybernétique.

37-38 Février-juin 1954 « Le loisir ».

Pierre Ducassé : Les philosophes et le loisir.

Joffre DUMAZEDIER : Histoire moderne et fonction du loisir.

Bernard GuILLEmAIN : Loisir et valeur : I'intention sportive.

39-40 Juillet 54 - Jan. 55 «L'information ».

Louis Couffignal: Science et technique de l'information.

François PAYCHA : L'information en médecine.

41-42 Février-août 1955 Pierre DucASSÉ : Intelligence technique et culture ouverte.

43-44 Sept. 55 - Fév. 56 Louis Couffignal : Science et technique de l'information (2e partie).

45-46 Mars-juillet 1956 «Technique et philosophie ».

Robert VALLÉ : Un schéma observationnel.

André Vergez : Réflexions sur les techniques de séparation et d'union de

l'âme et du corps chez Descartes.

47-48 Août-oct. 1956 Paul RenAud : Théorie de l'identification expérimentale.

Robert VAlkÉ : Quelques thèmes à tendance scientifique chez Edgar Allan Poe.

49-50 Nov. 56 - Jan. 57 Pierre Ducassé : Perspectives contemporaines sur l'œuvre d'Auguste Comte.

51-52 Février-avril 57 Lovis Couffignal : La cybernétique (essai méthodologique).

53-54 Mai-juillet 1957 Paul RenAud : Introduction à l'invention rationnelle. 
55-56 Août-sept. 1957 Louis CoufFIGNAL : La cybernétique des machines (1e partie).

Pierre Ducassé : Auguste Comte et la philosophie des techniques.

57-58 Nov. 57 - Jan. 58 Lovis CouffIGNAL : La cybernétique des machines (2e partie).

Geneviève DESSENNE : Polygénèse, systèmes polygénérants, phénomènes polygénétiques.

59-60 Février-avril 1958 Pierre Ducassé et Robert ValléE : Grandeur, décadence et ténacité des thèmes cybernétiques.

André VILLIERS : Justification d'une technique (l'éclairage de la scène centrale).

61-62 Déc. 1959 Louis Couffignal : La formation de l'ingénieur pour l'économie de l'avenir.

\begin{tabular}{|c|c|c|}
\hline $\begin{array}{l}63-64 \\
65-66\end{array}$ & Mars 1960 & $\begin{array}{l}\text { Michel DufFIEUX: Information et bruitages dans le dessin des formes matérielles. } \\
\text { Pierre DuCASSÉ : Situation du philosophe dans le monde des techniques ( } 1^{\mathrm{e}} \text { partie). }\end{array}$ \\
\hline $67-68$ & Juin 1960 & $\begin{array}{l}\text { Pierre DuCASSÉ : Situation du philosophe dans le monde des techniques (2e } \\
\text { partie). }\end{array}$ \\
\hline
\end{tabular}

69-70 Sept. 1960 Développement et formation de la personnalité des jeunes techniciens et scientifiques.

(Compte-rendu du Colloque international de Münich, avril 1959).

71-72 Janvier 1961 Louis CouffIGnal: Humanisme ou science ? Un faux problème.

73-74 Avril-juin 1961 Denis Huisman et Georges Patrix : Réflexions sur l'esthétique industrielle.

75-76 Juillet-sept. 1961 Lovis Couffignal : Les connaissances mathématiques indispensables au chercheur physicien et à l'ingénieur (Résumé des rapports et débats du colloque de l'OECE, juillet-sept. 1961).

77-78 Oct. 61 Pierre Ducassé : L'art de s'exprimer.

79-80 Juin 1962 Adolphe JARRIGE : Les utilisations industrielles des cendres volantes et la recherche fondamentale.

Henri Goвцoт : Le rôle de l'Iran dans les techniques de l'eau.

81-82 Oct. 62 Paul Renaud : Limitation des dégâts causés par les cyclones tropicaux.

83-84 Février 1963 Paul ReNAUD : La méthode des transformations fractionnées fournit une localisation des causes dont on ne connaît que les effets.

- : Compléments au procédé destiné à limiter les dégâts effectués par certains cyclones tropicaux et autres vents destructeurs ( $2^{\mathrm{e}}$ partie).

85-86 Mars 1963 Pierre Ducassé : Problèmes spécifiques de la «pédagogie des adultes ॥. Paul Renaud : Spécialités orthogonales à la classification des sciences.

87-88 Mai 1964 Michael BALINT : Comment préparer les praticiens de Médecine générale et les étudiants en médecine à s'acquitter de leur rôle dans le domaine de la santé mentale? 


\section{Textes de Pierre Ducassé (liste indicative)}

\section{Articles :}

"Le témoignage d'Auguste Comte et le réveil des espérances positives », Revue Positiviste Internationale, $31^{e}$ année, nov. 1936, nº 5-6.

"Le «temps» des techniques", Thalès, Recueil annuel des travaux de l'Institut d'histoire des sciences et des techniques de l'Université de Paris, tome V, 1948.

" Pour l'histoire de la mesure du temps », Thalès, Recueil annuel des travaux de l'Institut d'histoire des sciences et des techniques de l'Université de Paris, tome V, 1948.

«Intelligence technique et culture ouverte », S.É.T. n 41-42, 1955.

« Auguste Comte et la philosophie des techniques», S.É.T. n 55-56, 1957.

(avec Robert VAlḱE) « Grandeur, décadence et ténacité des thèmes cybernétiques 》, S.É.T n 59-60, 1958

"Situation du philosophe dans le monde des techniques» (en deux parties), S.É.T nº 63 à 68, 1960.

\section{Ouvrages :}

Essai sur les origines intuitives du positivisme, Paris, Alcan, 1939.

Méthode et intuition chez Auguste Comte, Paris, PUF, 1939.

Histoire des techniques, Paris, PUF, 1945 (4e éd. 1958), collection « Que sais-je ? » n 126.

Les techniques et le philosophe, Paris, PUF, 1958, collection « Philosophie de la matière ».

\section{Autres sources :}

Lettre de P. Ducassé à Paul Edger, 29 juin 1938, archives de la Maison d'Auguste Comte.

Pierre Ducassé, Curriculum Vitae, Archives du Cnam, Paris.

Rapport de séance du 4 juin 1956 du Conseil de perfectionnement du Cnam, Archives du Cnam, Paris. "Information: Une Association pour l'étude des techniques", Thalès, Recueil annuel des travaux de I'Institut d'histoire des sciences et des techniques de l'Université de Paris, tome V, 1948.

«L'association S. É. T. pour l'étude des techniques », S.É.T. n 1, pp. 1-2, 1948.

Robert Vallée : «Pierre Ducassé (1905 - 1983) », notice nécrologique, Bulletin d'information de l'AFCET Systémique, 17 avril 1984.

Entretien avec Jean-Pierre Ducassé (Maître de conférences honoraire au CNAM, fils de P. Ducassé) , 19 avril 2006.

Remerciements (par ordre alphabétique) :

Jean-François Braunstein, Lise Cloître, Jean-Pierre Ducassé, Claudine Fontanon, Aurélia Giusti, Robert Vallée. 